\title{
Bacterial translocation and intestinal injury in experimental necrotizing enterocolitis model
}

\author{
Ciftci $\mathrm{I}^{1}$, Ozdemir $\mathrm{M}^{2}$, Aktan $\mathrm{M}^{3}$, Aslan $\mathrm{K}^{4}$ \\ Department of Pediatric Surgery, Selcuk University, Selcuklu Medical Faculty, Konya, Turkey. driciftci@yahoo.com
}

\begin{abstract}
Objective: To study the occurrence of bacterial translocation and to assess the impact of breastfeeding on bacterial translocation in the animal model of necrotizing enterocolitis.

Methods: A total of 20 neonate Sprague-Dawley rats were enrolled in the study. Rats were randomly allocated into either control or study group just after birth. Ten newborn rats in the control group were left with their mother to be breast-fed. In contrary, necrotizing enterocolitis group consisted of neonates that were separated from their mothers, housed in an incubator and were gavaged with a special rodent formula three times daily. Survival rates, weight changes, and morphologic scoring obtained after microscopic evaluation were determined as microbiologic evaluation criteria.

Results: All the rats in the control group survived, while $1(10 \%)$ rat died in the necrotizing enterocolitis group. Mortality rates of the two groups were similar. All the formula-fed animals in the necrotizing enterocolitis group had significant weight loss compared to the breast milk-fed rats in the control group $(p<0.05)$. A total of $7(70 \%)$ and $2(20 \%)$ E. coli growths were identified in the bowel lumen, liver, and spleen of necrotizing enterocolitis and control groups, respectively. This difference was statistically significant. In peritoneal smear cultures, a total of $3(30 \%)$ growths were detected in the necrotizing enterocolitis group and $1(10 \%)$ growth in the control group. Conclusion: As the result of a disturbance in the intestinal flora and impairment of the intestinal barrier in necrotizing enterocolitis, microrganisms in the bowel pass through the intestinal barrier and reach the liver and the spleen via the hematogenous route. This condition is closely related to the impairment of physiological and functional features of the intestinal barrier and is independent from the degree of intestinal injury. Bacterial translocation should be remembered in cases suspected of necrotizing enterocolitis, and a rapid and effective treatment algorithm should be applied in such circumstances (Tab. 3, Fig. 3, Ref. 21). Full Text in PDF www.elis.sk. Key words: necrotizing enterocolitis, bacteria, translocation, epithelial barrier, mucosa, maternal milk feedings.
\end{abstract}

Necrotizing enterocolitis (NEC) is the most common surgical emergency among newborns and premature infants in pediatric surgery clinics and has a higher mortality than other gastrointestinal system (GIS) diseases requiring surgery (1). Its incidence has gradually increased in the last decade. Mortality rate varies between $10-50 \%$ and is higher in cases of pannecrosis (2). Etiology of this severe disease could not been explained thoroughly despite clinical and laboratory investigations.

Etiopathogenesis of NEC has not been fully understood. Prematurity leading to an intestinal immaturity may be one of the predisposing factors. Other possible causes are undeveloped new-

${ }^{1}$ Department of Pediatric Surgery, Selcuk University, Selcuklu Medical Faculty, Konya, Turkey, ${ }^{2}$ Department of Microbiology and Clinical Microbiology, Selcuk University, Meram Medical Faculty, Konya, Turkey, ${ }^{3}$ Department of Histology and Embryology, Selcuk University, Meram Medical Faculty, Konya, Turkey, and ${ }^{4}$ Department of General Surgery, Konya Research and Training Hospital, Konya, Turkey

Address for correspondence: I. Ciftci, MD, Selcuk Universitesi Selcuklu Tip Fakultesi, Cocuk Cerrahisi A.D. Konya, Turkey.

Phone: +903322415000, Fax: +903322412184

Acknowledgement: This manuscript presented in "The World Federation of Associations of Pediatric Surgeons (WOFAPS) annual meeting Updates in Pediatric Surgery: Controversies and Advances-2011" born intestinal barrier, hypersensitive splanchnic hypoperfusion, intestinal injury due to disturbance of mesenteric circulation in perinatal asphyxia, and invasion of microflora (3).

Studies have indicated that breast milk has a protective role in all infections and NEC (4). Breast milk provides secretory IgA and macrophage for the baby, and incidence of NEC is reported to be lower among breast-fed babies. Breast milk also provides intestinal flora of the newborn to be colonized by bifidobacterium and reduces pathogen colonization (5).

Necrotizing enterocolitis has been suggested to be an infectious disease caused by various microorganisms involving normal or injured intestine (6). The role of infection in the pathophysiology of NEC has been widely studied in literature. In a study on infants with NEC, many microorganisms like ECHO virus type 22, Escherichia coli (E. coli), Klebsiella have grown in the blood, feces and CSF cultures (7). The presence of hydrogen (in $30 \%$ cases) and pneumatosis intestinalis appearance indicates the role of bacteria in etiopathogenesis (8). Responsiveness to antibiotics also supports the infectious pathogenesis.

Another evidence for infectious etiology in NEC is the development of NEC-like lesions in the experimental administration of endotoxins - Endotoxins mediate Tumor Necrosis Factor- $\alpha$ (TNF- $\alpha$ ) and Platelet Activating Factor (PAF). Levels of TNF- $\alpha$, 
PAF and interleukin- 6 levels are found to be high in the sera of premature infants with NEC. Endotoxin has been detected in $80 \%$ of cases in whose blood culture gram negative bacteria has grown and in approximately $50 \%$ of all NEC cases (9).

An impaired intestinal barrier is an important factor for the development of NEC progression of the disease. Intestinal barrier is a functional and anatomic defense system preventing the body from the invasion of enteric bacteria. Factors constituting the intestinal barrier are gastric acid, pepsin, pancreatic enzymes, mucus, immunoglobulines and intestinal peristaltics.

One of the most commonly accused etiologic factors is the reduction of mucosal perfusion that subsequently leads to the hypoxia of the mucosa, cell injury and necrosis. Proper conditions for the bacterial invasion is formed when the anatomic barrier is destroyed due to the mucosal necrosis and impairment of the intestinal mucosal maturity $(10,11)$.

The aim of this study was to study the affect and degree of intestinal injury of bacterial translocation on necrotizing enterocolitis.

\section{Methods}

Experimental Study: Pregnant time-dated Sprague-Dawley rats were induced at term using subcutaneous injection of pitocin (1 U). Immediately after birth, the neonates were weighed and randomized into one of two groups. Group 1 (control group) consisted of 10 neonatal rats that were left with their mother and thus were breast-fed. Group 2 (NEC group) consisted of 10 neonates that were separated from their mothers, housed in an incubator and were gavaged with a special rodent formula three times daily as described by Nadler et al (12). The formula consisted of $15 \mathrm{~g}$ Similac 60/40 (Ross Pediatrics. Columbus, OH) in $75 \mathrm{ml}$ of Puppymilk canine milk replacement (Beaphar-bogena, B.V. Sedel, Nederland) as described by Barlow and was designed to approximate the protein and caloric content of the rat breast milk (13). Induction of experimental NEC was performed by exposing the subjects to asphyxia (breathing $100 \%$ nitrogen gas for $60 \mathrm{~s}$ ) and cold stress $\left(4{ }^{\circ} \mathrm{C}\right.$ for $10 \mathrm{~min}$ ) twice daily. The neonatal rats were weighed and sacrificed on the day 4: Peritoneal smear culture, pieces from spleen, liver and $2 \mathrm{~cm}$ of intestine were obtained. Samples were obtained for culture from the opened lumen.

Assessment: Survival rates, weight changes, morphologic scoring obtained after microscopic evaluation were determined as microbiologic evaluation criteria.

Morphological studies: Intestinal specimens were fixed in 4\% formalin, embedded in paraffin; $(5-7 \mu)$ sections were stained with hematoxylin and eosin ( $H \& E$ ) for microscopic evaluation (4). The morphological changes in the intestinal epithelium were scaled according to Nadler et al, as normal, mild, moderate and severe by a histologist (MA), who was blinded to the treatment groups (12). The definition of each histological grade was as follows: (0) normal histological appearance; (1) mild, separation of the villus core, without other abnormalities; (2) moderate, villus core separation, submucosal edema, and epithelial sloughing; (3) severe, denudation of epithelium with loss of villi, full thickness necrosis, or perforation.
Microbiological study: Animals in all groups underwent laparatomy with anesthesia protocol and under sterile conditions. Liver and spleen were dissected for culture in all test subjects. One gram of samples were pounded in a sterile environment and homogenized in homogenizators so as to be diluted in the ratio of 1/10 in BHI medium. $0.01 \mathrm{ml}$ of homogenized suspension was taken with urine and inoculated in the blood agar, EMB agar and $5 \%$ sheep blood Schaedler agar. Cultures in blood agar and EMB agar were inoculated at $37^{\circ} \mathrm{C}$ in aerobe medium and cultures in Schaedler agar were inoculated at $37^{\circ} \mathrm{C}$ under anaerobic conditions.

Procedures performed for liver and spleen samples were repeated for ileum. Growing microorganisms were analyzed in terms of colony morphologies in hard cultures, pigment and hemolysis formation, Gram staining method and morphological characteristics. Routine biochemical tests were used for identification. In order to identify gram negative enteric bacteriae, growing features, motion features, oxidase, catalase and indol formation, effects on urea, citrate, arginin, lysine, ornithine and various carbohydrates in triple sugar iron (TSI) culture were studied. For identification of Gram positive bacteriae, oxidation and fermentation making, plasma coagulase and catalase formation, effects on mannitol, susceptibilities to novobiocin, bacitracin and optokin were investigated. Numbers of colonies of growing microorganisms were counted and the results were recorded by multiplying with 1000 .

Statistical analysis: Changes in the weight were calculated according to the formula: [(first weight - last weight)/ first weight $\mathrm{X} 100]$. The Mann Whitney U test and Chi-square test were used to compare the parametric and categorical data of the two groups respectively. The level of significance was taken as $\mathrm{p}<0.05$.

\section{Results}

All rats in the control group had survived, while one of the rats $(10 \%)$ died in the NEC group. Mortality rates of two groups were similar. All formula-fed animals in the NEC group had a significant weight loss compared to the breast milk-fed rats in the control group (Tab. 1) (Fig. 1).

Diagnosis of NEC was confirmed macroscopically (Fig. 2). Morphologic analysis of the intestinal segments from the breastfed animals showed no significant histological abnormalities. The histopathological changes were scored into 4 categories ranging from Score 0: normal architecture to Score 4: frank bowel necro-

Tab. 1. Weight changes in groups.

\begin{tabular}{clccc}
\hline Group & & Initial weight & Recent weight & Weight change \\
\hline 1 & Mean & 5.7800 & 6.9310 & 19.8279 \\
& $\mathrm{~N}$ & 10 & 10 & 10 \\
& Std. deviation & .25495 & 1.34115 & 22.68243 \\
\hline 2 & Mean & 5.6330 & 4.0444 & -27.8009 \\
& $\mathrm{~N}$ & 10 & 9 & 9 \\
& Std. deviation & .31330 & .47886 & 11.03763 \\
\hline Total & Mean & 5.7065 & 5.5637 & -2.7331 \\
& $\mathrm{~N}$ & 20 & 19 & 19 \\
& Std. deviation & .28805 & 1.78716 & 30.13912 \\
\hline
\end{tabular}




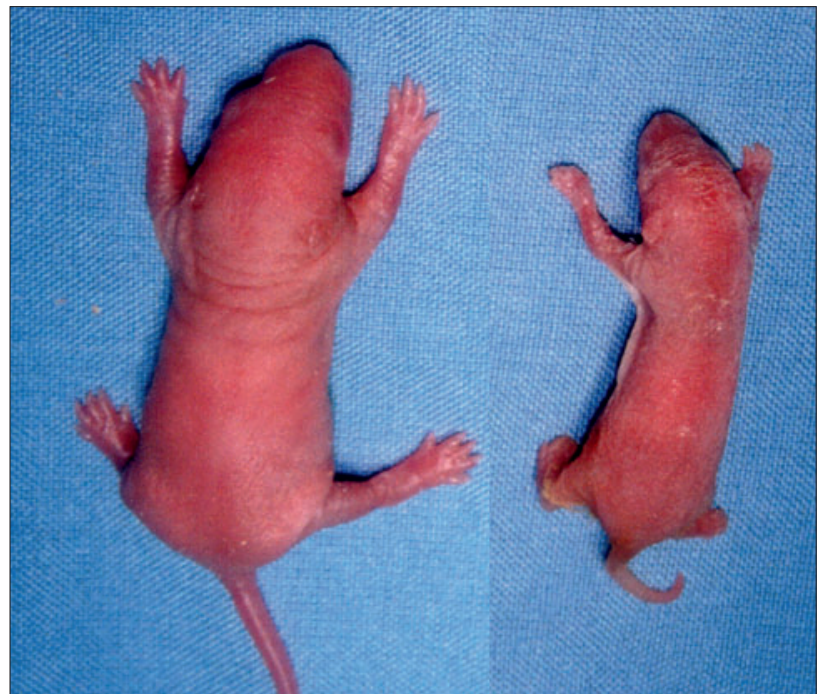

Fig. 1. Weight changes in groups.

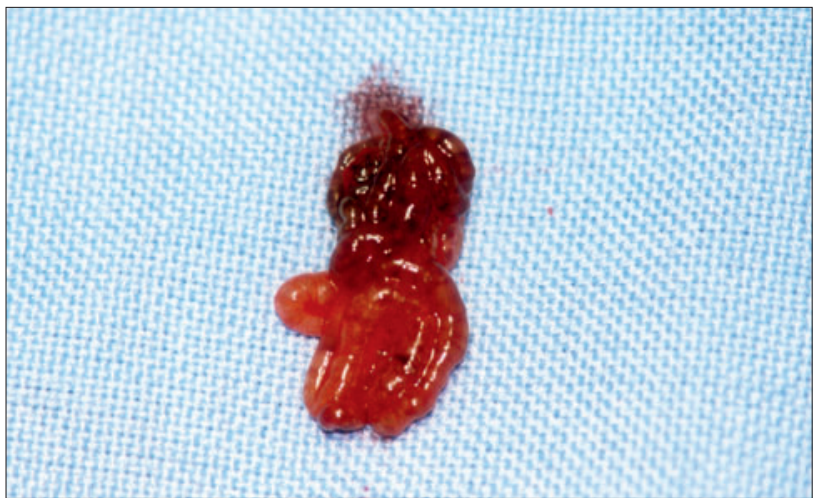

Fig. 2. NEC was detected macroscopically.

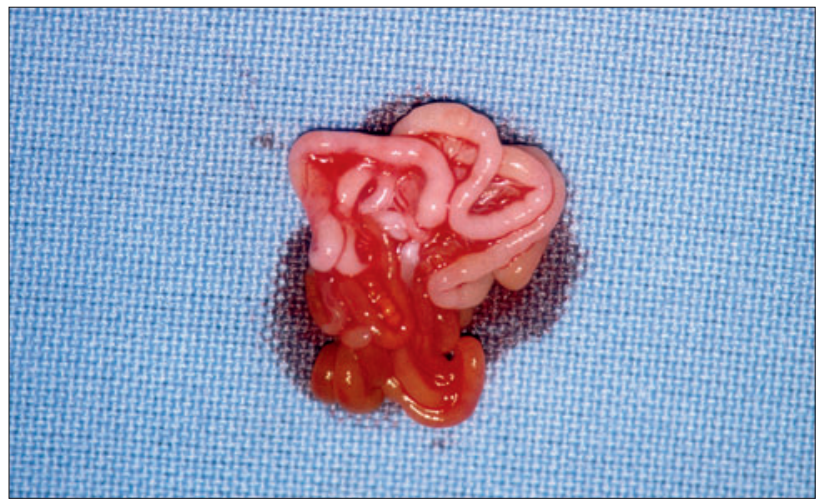

Fig. 3. Normal intestinal architecture.

sis. All the breast-fed animals had normal intestinal architecture in the control group (Fig. 3). In contrast, the animals in the NEC group showed various degrees of inflammatory changes ranging from epithelial sloughing (Score: 1) to frank bowel necrosis (Score: 3). All these animals had moderate or severe histological abnormalities (Tab. 2).
Tab. 2. Histological abnormalities.

\begin{tabular}{|c|c|c|c|c|c|c|c|}
\hline & & & \multicolumn{4}{|l|}{ Score } & \multirow{2}{*}{ Total } \\
\hline & & & 0 & 1 & 2 & 3 & \\
\hline \multirow[t]{4}{*}{ Group } & 1 & number & 10 & 0 & 0 & 0 & 10 \\
\hline & & $\%$ group 1 & $100 \%$ & $0 \%$ & $0 \%$ & $0 \%$ & $100 \%$ \\
\hline & 2 & number & 2 & 3 & 3 & 1 & 9 \\
\hline & & $\%$ group 2 & $22.2 \%$ & $33.3 \%$ & $33.3 \%$ & $11.1 \%$ & $100.0 \%$ \\
\hline
\end{tabular}

Tab. 3. Bacterial translocation in NEC and control group.

\begin{tabular}{|c|c|c|c|c|c|}
\hline & & & lumen & & \\
\hline & & & growing (-) & growing $(+)$ & lotal \\
\hline$\overline{\text { Group }}$ & 1 & number & 2 & 8 & 10 \\
\hline & 2 & number & 0 & 9 & 9 \\
\hline & & & peritoneal smea & & total \\
\hline & & & growing (-) & growing $(+)$ & total \\
\hline$\overline{\text { Group }}$ & 1 & number & 9 & 1 & 10 \\
\hline & 2 & number & 6 & 3 & 9 \\
\hline & & & liver & & total \\
\hline & & & growing (-) & growing $(+)$ & total \\
\hline Group & 1 & number & 8 & 2 & 10 \\
\hline & 2 & number & 5 & 4 & 9 \\
\hline & & & spleen & & total \\
\hline & & & growing (-) & growing $(+)$ & total \\
\hline Group & 1 & number & 8 & 2 & 10 \\
\hline & 2 & number & 2 & 7 & 9 \\
\hline
\end{tabular}

Microbiologic analysis: In the NEC group, a total of 7 (70 \%) growths were detected, mainly E. coli in lumen, liver and spleen. Two $(20 \%)$ growths were detected in the control group. This difference was statistically significant. In the peritoneal smear culture, a total of 3 (30\%) growths were detected in the NEC group and $1(10 \%)$ growth in the control group. A statistically significant difference was not seen (Tab. 3).

\section{Discussion}

At birth, there is a gastrointestinal flora including more than 500 species and $10^{14}$ bacteriae, exhibiting variations individually for each person in terms of birth type, genetic features, environmental contact, and nutrition type. This microflora especially in colon and distal intestine has a crucial biologic activity (1-3).

These commensal bacteriae provide significant benefits on nutrition, angiogenesis and mucosal immunity. Intestinal bacteriae play roles on bile salt metabolism, lipid hydrolysis, degradation of proteins to peptide and aminoacids and vitamin production $(2,3)$.

The immaturity of gastrointestinal tract immunity makes the neonatal GI tract susceptible to colonization of pathogenic bacteria. Some postulates supporting the theory of abnormal bacterial colonization are as follows: First, NEC usually exists in the second week after birth, after the floral colonization of neonatal intestine. Second, NEC usually occurs sporadically. Despite the reported outbreaks in crowded nurseries, these outbreaks have never been fully attributed to a specific infectious microorganism. Therefore, the bacteria contributing to the development of NEC are actually normal inhabitants of the intestine rather than pathogenic organisms transmitted from one infant to another. The intestinal flora 
plays an important role in both maintaining the intestinal barrier, and dampening the inflammatory response. Vulnerability of premature infants' ensource from two causes: (i) exposure to various nosocomial bacteria in the neonatal ICU; (ii) immaturity of their immune systems, which do not respond appropriately to normal bacterial colonization (15).

The immaturity of intestinal epithelial cells in the premature infant is another contributor to the NEC pathogenesis. The integrity of the premature infant's intestinal barrier is compromised because premature babies do not yet have a developed coordinated peristalsis. The prolonged exposure of the intestinal epithelium to potentially noxious substances lead to formation of immature mucous coats and improperly formed tight junctions. Eventually, intestinal permeability is increased and the regeneration of the intestinal barrier is impaired (15).

A developed defense system is established in gastrointestinal system with wide mucosal surface area against nutritional antigens, pathogen microorganisms and environmental agents via intestinal epithelium barrier, intestinal mucosal maturity, mucosal immune system and intestinal microflora (1-3). Bacteriae and antigens' passing from intestinal lumen to circulation is hindered with mucus barrier on epithelial surface and tight connections between epithelial cells. An important component of the barrier function is mucosal immune system. Gut associated lymphoid tissue, (GALT), is the greatest immune organ in the body involving $70-80 \%$ of the all immune cells in the body that are mostly lymphocytes located in Peyer Plaques. Antigens in the intestinal lumen are taken with special epithelial cells on Peyer Plaques (M cells) and presented for later education of CD4+ regulator T cell populations (Treg) after being processed in the local dendritic cells. Tolerance to the antigen is established by the recognition of antigen samples of the normal flora that leads to the release of cytokines such as IL-10, TGF- $\beta$ that in turn would suppress the effector lymphocytes. Gut associated lymphoid tissue is the most important defense organ for both congenital and acquired immunity against potential pathogens (16).

Gut associated lymphoid tissue is hypoplastic in cases having no or impaired intestinal colonization. Reduction of the normal flora by infection or antibiotics negatively affects gastrointestinal mucosa development and formation of natural and acquired immunity responses.

Bacterial translocation is known as passing alive bacteriae and their products in intestinal system to lymph nodes and systemic circulation. It easily develops when mucosal barrier is impaired, pathologic changes develop in mucus secretion, peristaltic activity and intestinal flora, immunity system weakens $(1,2)$. Factors such as hypoxia, immaturity, and impairment of flora may facilitate the bacterial translocation and contribute to the pathogenesis of $\operatorname{NEC}(17,18)$.

Breast milk is known to have immune properties as it includes specific immunoglobulin A, lysozyme and lactoferrin. Therefore breast milk is thought to protect the preterm infant from bacterial and viral infections and enteral breast milk feeding for very low birth weight infants reducing the risk of necrotising enterocolitis (19).
In this study, we detected that bacterial translocation developed consistently with the GIS flora that was reported to be isolated simultaneously in the liver and spleen cultures of the subjects (20). Floral microorganisms in the control group that were not isolated from liver and spleen indicates the absence of translocation. These results indicate that bacterial translocation has also occurred in our NEC model. Culture results revealed the growth of bacteriae like E. coli, Proteus, Enterobacter, Staphylococcus and Klebsiella.

One of our most noteworthy findings is that isolation of microorganisms in liver and spleen occurred independently from the degree of intestinal injury. This may result from the impairment of intestinal barrier, which can no more prevent bacterial translocation. Analysis of our results indicated that microorganisms isolated in the liver and the spleen could not be isolated in the peritoneal smear. This result signifies that a disturbance of the barrier function may happen without the complete loss of intestinal integrity and translocation may in turn develop this setting. Development of a systemic infection is enhanced as a consequence of this translocation (21).

\section{Conclusion}

In conclusion, disturbance of intestinal flora and impairment of the intestinal barrier are important contributors for pathogenesis of NEC. Passage of microorganisms through the intestinal barrier is followed by invasion of liver and spleen via hematogenous route. In this circumstance, malfunction of intestinal barrier is independent from the degree of intestinal injury. Possibility of the impairment of the barrier and bacterial translocation must be kept in mind in cases suspected of NEC. This condition should be taken into consideration not only in diagnostic terms but also for the determination of an effective treatment strategy.

\section{References}

1. Kliegman RM, Panaroff AA. Necrotizing enterocolitis. N Engl J Med 1984; 310: 1093-1103.

2. Rowe MI, Reblock KK, Kurkchubasche AG et al. Necrotizing enterocolitis in the extremely low birth weight infant. J Pediatr Surg 1994; 29: 987-990.

3. Sheard NF, Walker WA. The role of breast milk in the development of gastrointestinal tract. Nutr Rev 1988; 46: 1-8.

4. Dickinson EC, Gorga JC, Garrett M et al. Ig A supplementation abrogates bacterial translocation and preserves the architecture of the intestinal epithelium. Surgery 1998; 124: 284-290.

5. Yoshioka H, Iseki K, Fujita K. Development and differences of intestinal flora in the neonatal period in tha breast-fed and bottle-fed infants. Pediatrics 1983; 72: 317-321.

6. Kosloska AM, Ulrich JA. A bacteriologic basis for the clinical presentation of necrotizing enterocolitis. J Pediatr Surg 1980; 15: 558-564.

7. Rotbart HA, Levin MJ. How contagious is necrotizing enterocolitis? Pediatr J Infect Dis 1983; 2: 406.

8. Engel RR, Modler S, Matsen JM et al. Carbon monoxide production from hydroxocobalamin by bacteria. Biochim Biophys Acta 1973; 313: $150-155$. 


\section{6-210}

9. Kosloske AM, Musemeche CA. Necrotizing enterocolitis of the neonate. Clin Perinatol 1989; 16: 97-111.

10. McElhinney DB, Hedrick HL, Bush DM et al. Necrotizing enterocolitis in neonates with congenital heart disease: risk factors and outcomes. Pediatrics 2000; 106: 1080-1087.

11. Grosfeld JL, Cheu H, Schlatter $M$ et al. Changing trends in necrotizing enterocolitis. Experience with 302 cases in two decades. Ann Surg. 1991; 214: 300-306; discussion 306-307.

12. Nadler EP, Dickinson E, Knisely A et al. Expression of inducible nitric oxide synthase and interleukin-12 in experimental necrotizing enterocolitis. J Surg Res 2000; 92: 71-77.

13. Barlow B, Santulli TV, Heird WC et al. An experimental study of acut neonatal enterocolitis: The importance of breast milk, J Pediatr Surg 1974: 9; 587-595.

14. Dvorak B, Halpern MD, Holubec $\mathbf{H}$ et al. Epidermal growth factor reduces the development of necrotizing enterocolitis in a neonatal rat model. Am J Physiol Gastrointest Liver Physiol 2002; 282: G156-G164.

15. Berman L, Moss LR. Necrotizing entrocolitis: an update. Semin Fetal Neonatal Med 2011; 16: 145-150.
16. Anand RJ, Leaphart CL, Mollen KP et al. The role of the intestinal barrier in the pathogenesis of necrotizing enterocolitis. Shock 2007; 27: $124-133$

17. Clark DA, Thompson JE, Weiner LB et al. Necrotizing enterocolitis: Intraluminal biochemistry in human neonates and rabbit model. Pediatr Res 1985; 16: 919-921.

18. Anand RJ, Leaphart CL, Mollen KP et al. The role of the intestinal barrier in the pathogenesis of necrotizing enterocolitis. Shock 2007; 27: $124-133$.

19. Chauhan M, Henderson G, McGuire W. Enteral feeding for very low birth weight infants: reducing the risk of necrotising enterocolitis. Arch Dis Child Fetal Neonatal Ed. 2008: 93: F162-166.

20. Jackson RJ, Smith SD, Wadowsky RM et al. The effect of E coli virulence on bacterial translocation and systemic sepsis in the neonatal rabbit model. J Pediatr Surg 1991; 26: 483-485; discussion 485-486.

21. Sherman MP. New concepts of microbial translocation in the neonatal intestine: mechanisms and prevention. Clin Perinatol 2010; 37: 565-579.

Received November 17, 2011. Accepted November 26, 2011. 